\title{
New fabrication techniques for high quality photonic crystals
}

\author{
Chuan C. Cheng ${ }^{\mathrm{a})}$ and Axel Scherer \\ Electrical Engineering, California Institute of Technology, Pasadena, California 91125
}

\author{
Rong-Chung Tyan and Yeshayahu Fainman \\ Electrical and Computer Engineering, University of California, San Diego, La Jolla, California 92093
}

George Witzgall and Eli Yablonovitch

Electrical Engineering, University of California, Los Angeles, Los Angeles, California 90095

(Received 30 May 1997; accepted 6 August 1997)

\begin{abstract}
We have developed new methods for the fabrication of high quality two-dimensional (2D) and three-dimensional (3D) photonic crystals. These techniques involve anisotropic etching and steam oxidation of AlAs mask layers. We have made manufacturable 2D photonic crystals with high aspect ratios for use as micropolarizers and have measured extinction ratios larger than 800 to 1 between TE and TM modes transmitted through these structures. The new $\mathrm{Al}_{2} \mathrm{O}_{3}$ mask fabrication technique also allows us to fabricate 3D structures with up to six repeating layers in depth and over $90 \%$ attenuation in the band gap region. Here, we show the fabrication details and performance of 2D and 3D photonic crystals. (C) 1997 American Vacuum Society. [S0734-211X(97)12006-6]
\end{abstract}

\section{INTRODUCTION}

The properties of a photon can be modified in a periodic dielectric structure if the period of the structure is comparable to the wavelength of light. In the one-dimensional case, we are familiar with the quarter wave stack or Bragg reflector. When light is propagating perpendicular to this structure, the reflectivity spectrum shows a high reflectivity region or one-dimensional (1D) stop band. If the structure has periodicity in all directions, a complete three-dimensional (3D) photonic band gap can open in all directions. Photonic band gap structures are expected to lead to devices with interesting and unusual properties such as inhibition of spontaneous emission and localization of modes. ${ }^{1-7}$ Originally, the application for photonic crystals thus were focused on lowering threshold currents for lasers and increasing the efficiency of light emitting diodes. ${ }^{7}$ However, more recently passive elements such as high reflectivity mirrors and polarizing beam splitters have also become very attractive.

Many groups have successfully designed and measured two-dimensional (2D) and 3D photonic structures in the microwave region. ${ }^{4,5}$ However, because of the absence of easy microfabrication processes, the interest in using photonic crystals in the optical region has focused mainly on 1D and 2D photonic crystals. ${ }^{8,9}$ In the past, 2D photonic crystals have been made by planar pattern definition of rods or holes perpendicular to the wafer surface, by transferring $2 \mathrm{D}$ periodic patterns straight into semiconductor. This pattern transfer relies on high aspect ratios or on very thin, strongly confined waveguides to reasonably approximate ideal 2D geometries. Here, we demonstrate an easy alternative way to generate high quality 2D photonic crystals in which the structure which defines the $2 \mathrm{D}$ photonic crystal lies in the plane of the surface of the devices.

We are also interested in 3D crystals based on a fcc-like structure with three straight holes etched into a semiconduc-

$\overline{{ }^{a} \text { Electronic mail: cccheng@cco.caltech.edu }}$ tor surface. ${ }^{4}$ We have previously reported the successful fabrication of such crystals. ${ }^{10,11}$ The main difficulty of building this crystal lies in finding a robust etch mask. In this article, we will present our latest 3D fabrication techniques for fabricating these crystals.

\section{FABRICATION}

\section{A. 2D photonic crystals}

Typically, drilling a set of holes straight into semiconductor forms a 2D photonic crystal. For good performance of the photonic crystal, the 2D photonic structure must have a high aspect ratio in the $Z$ direction, which is the direction normal to the incident plane. The technology for building these crystals into semiconductor waveguides requires highly anisotropic etching of very small structures.

We have developed another, more manufacturable method to fabricate 2D photonic crystals in which the $Z$ direction of the 2D photonic crystal lies parallel to the surface of the semiconductor. Figure 1 shows the fabrication procedures of these two different structures. The new geometry allows easy fabrication of high aspect ratio $2 \mathrm{D}$ photonic crystals, which are quasi-infinite in the length, by etching a line grating through a 1D Bragg reflector. The number of repeating periods is, in this case, determined by the initial layer structure previously grown on the semiconductor. First, five $\mathrm{Si} / \mathrm{SiO}_{2}$ alternate layers with $130 / 260 \mathrm{~nm}$, respectively, are deposited by rf sputter deposition on the top of a glass substrate. Then, electron beam lithography defines a $300 \mathrm{~nm}$ line grating with a $600 \mathrm{~nm}$ period in a $200 \mathrm{~nm}$ thick electron beam resist, polymethylmethacrylate (PMMA), layer. This PMMA layer then serves as a template to lift off $100 \mathrm{~nm}$ of chromium. The chromium layer in turn is the etch mask during the sequential $\mathrm{Si}$ and $\mathrm{SiO}_{2}$ reactive ion etching process. We use $\mathrm{C}_{2} \mathrm{~F}_{6}$ at $30 \mathrm{mTorr}$ and $50 \mathrm{~W}$ for $10 \mathrm{~min}$ to etch through $\mathrm{SiO}_{2}$ layers and a mixture of $\mathrm{NF}_{3} / \mathrm{CCl}_{2} \mathrm{~F}_{2}$ at $150 \mathrm{~W}$ and $20 \mathrm{mTorr}$ for $1.5 \mathrm{~min}$ to etch the Si layers. Finally, the 

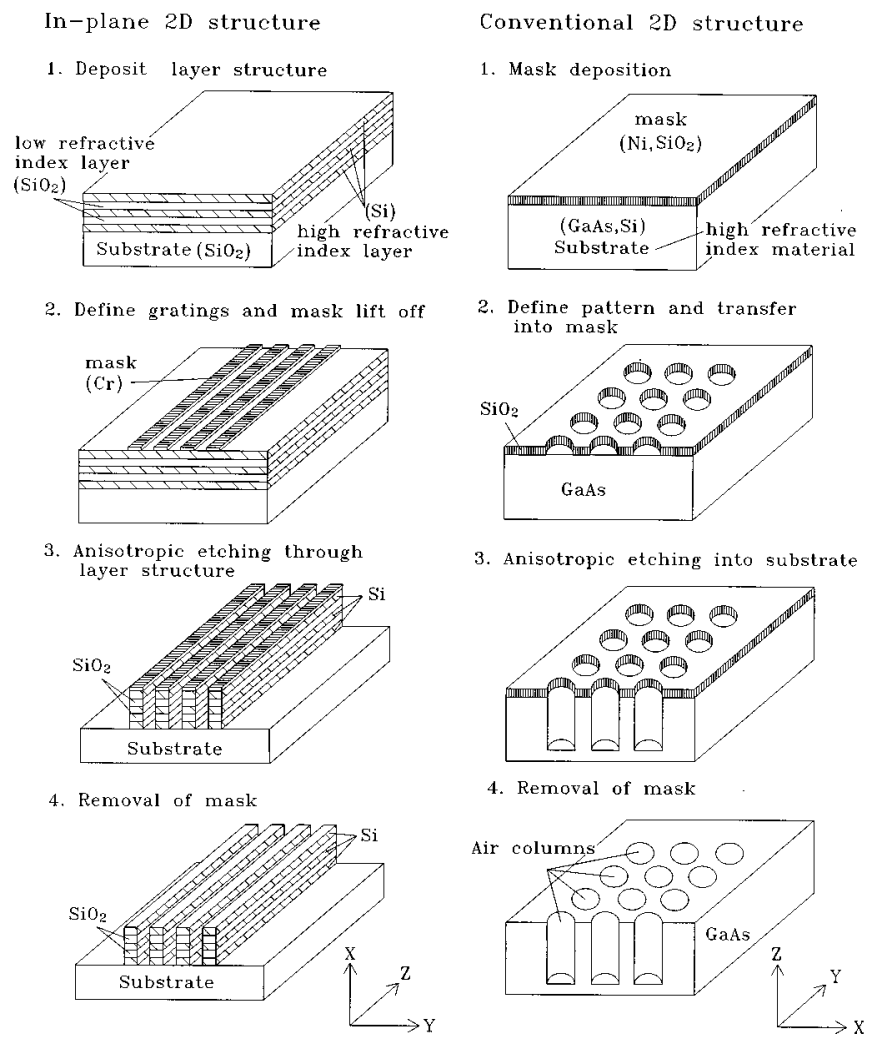

FIG. 1. Fabrication procedures of conventional and developed 2D photonic crystals.

remaining chromium mask is removed by a wet etch. In this way, a high quality photonic crystal with $600 \mathrm{~nm}$ period and $0.9 \mu \mathrm{m}$ deep is constructed. Figure 2 shows a scanning electron micrograph of a $2 \mathrm{D}$ photonic crystal etched through a layered structure.

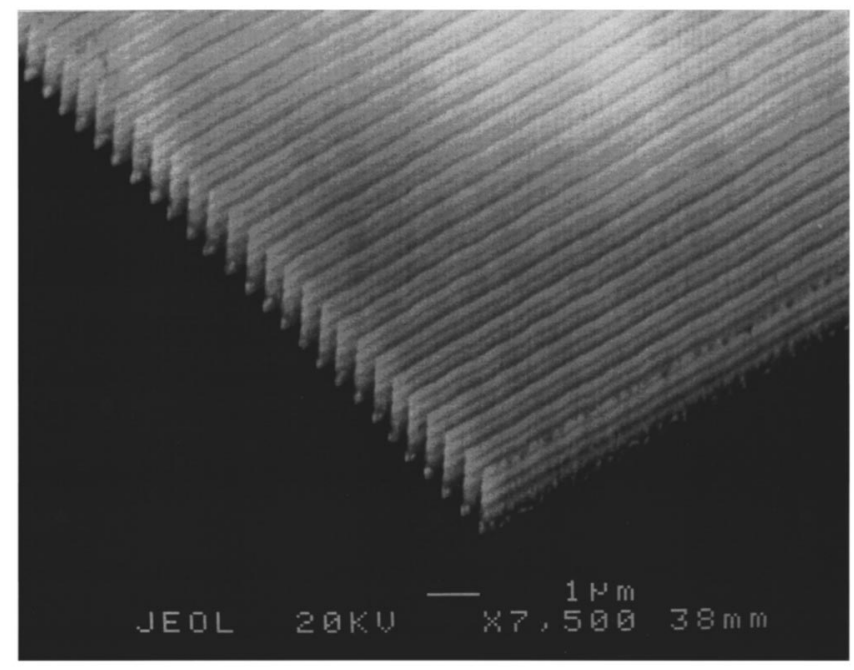

FIG. 2. Scanning electron micrograph of a $2 \mathrm{D}$ photonic crystal etched through a layered structure.
1. MBE growth structure

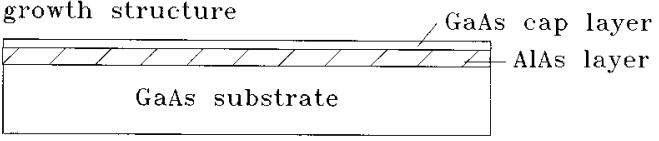

2. Mask deposition

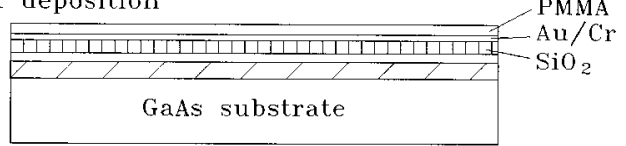

3. Ebeam lithography

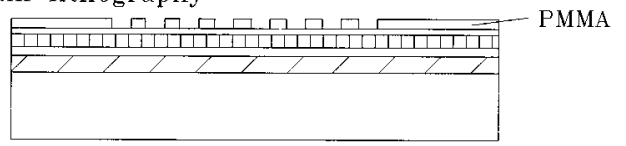

4. $\mathrm{Ar} / \mathrm{Cr}$ ion milling

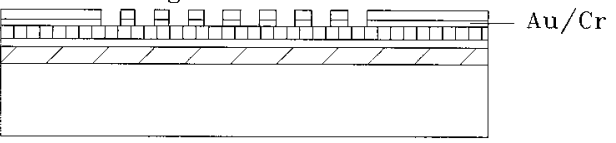

5. $\mathrm{SiO}_{2}$ reactive ion etching

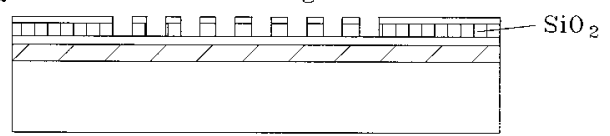

6. Angle ion beam etching through AlAs layer

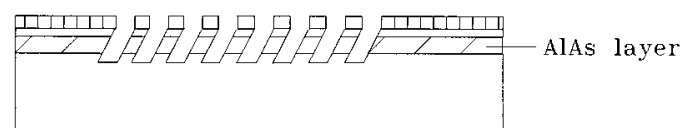

7. Steam oxidation converts $\mathrm{AlAs}$ to $\mathrm{Al}_{2} \mathrm{O}_{3}$

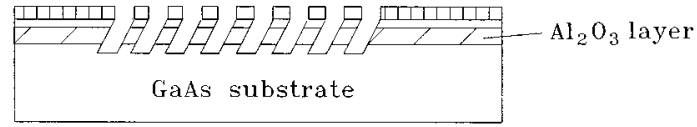

8. Transfer pattern into semiconductor in three directions

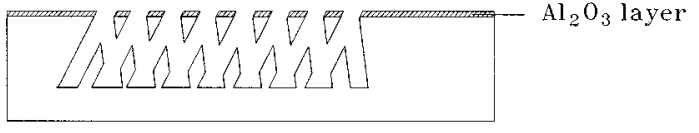

FIG. 3. Fabrication procedure of 3D photonic crystals.

\section{B. 3D photonic crystals}

The microfabrication of 3D photonic crystals is more difficult than that of 2D crystals because angle etching or regrowth are required to obtain a refraction index modulation in the $Z$ direction. We have previously reported the successful microfabrication of 3D photonic crystals with four repeating $(1,1,1)$ layers in depth fabricated for reflection in the infrared range. ${ }^{11}$ These previous results were obtained by using a robust planar $\mathrm{SiO}_{2}$ mask. The main difficulties of constructing these crystals with dramatically improved optical performance lie in the limited depth of the crystals, as well as the fidelity of mask replication. Usually, the finite selectivity of a planar mask severely limits the total depth of the crystal.

We have developed an alternative way for easier pattern transfer and for a higher selectivity between the etch rate of the mask and the semiconductor. Figure 3 shows the most recent fabrication procedure used for defining 3D photonic crystals. First, a $200 \mathrm{~nm}$ AlAs layer with a thin GaAs cap 


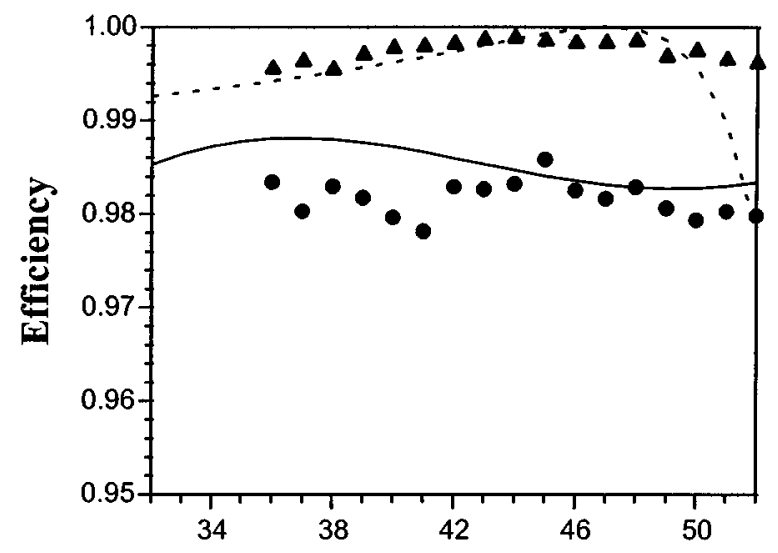

(a)

Incident angle (degree)

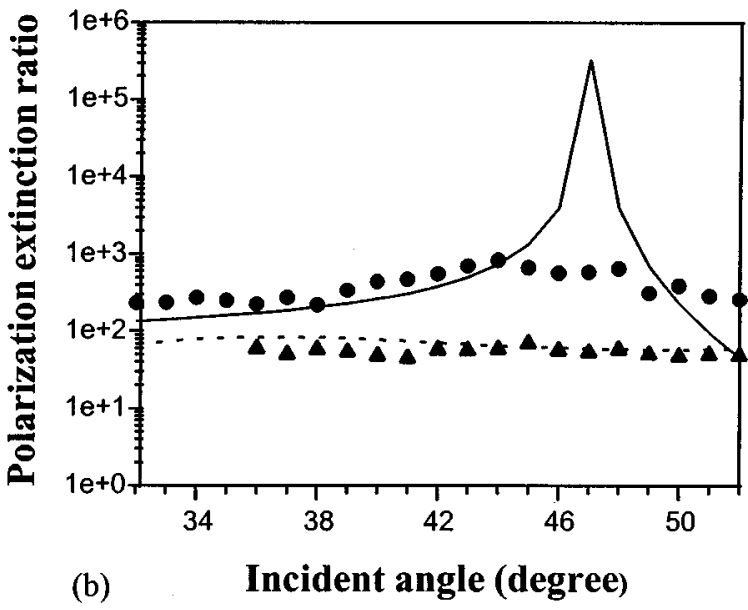

- Measured TM transmision - RCWA TM transmission

- Measured TE reflection ... RCWA TE reflection wavelength $\lambda=1.5 \mu \mathrm{m}$

FIG. 4. (a) Measured and calculated efficiencies for TE and TM polarized light vs incident angle at $1.5 \mu \mathrm{m}$. (b) Measured and calculated polarization extinction ratios in transmission and reflection vs incident angle at $1.5 \mu \mathrm{m}$.

layer are grown by molecular beam epitaxy (MBE) on the top of the GaAs substrate. The sample is then coated with $\mathrm{SiO}_{2}$ and $\mathrm{Au}$. Next, electron beam lithography defines a triangular array of circular holes which corresponds to the $(1,1,1)$ plane on PMMA. Then, this pattern is transferred into a 50-nm-thick $\mathrm{SiO}_{2}$ layer by $\mathrm{C}_{2} \mathrm{~F}_{6}$ reactive-ion etching. The $\mathrm{SiO}_{2}$ layer serves as an etching mask under $\mathrm{Ar} / \mathrm{Cl}_{2}$ ion beam assisted etching at a $35^{\circ}$ angle. ${ }^{12}$ The three-hole symmetric pattern is transferred into an AlAs layer which is subsequentially converted into $\mathrm{Al}_{2} \mathrm{O}_{3} \cdot{ }^{13}$ We have found that the $\mathrm{Al}_{2} \mathrm{O}_{3}$ layer has an etch rate selectivity over GaAs larger than 30 . This robust $\mathrm{Al}_{2} \mathrm{O}_{3}$ mask combined with directional-angle ion-beam etching can be used to define a deeper 3D photonic crystal. Care must be taken when etching into three directions since the holes are at $35^{\circ}$ angle to the surface normal. The first angle etch has to thin down the $\mathrm{Al}_{2} \mathrm{O}_{3}$ mask sufficiently to reduce any shadow effect for the other two directions. We have so far made photonic crystals with six repeating layers in depth by using such a $\mathrm{Al}_{2} \mathrm{O}_{3}$ angle-etch mask. This method gives us more freedom in designing the structure compared with our previous work using a $\mathrm{Ni}$ or $\mathrm{SiO}_{2}$ mask.

The simple pattern transfer into AlAs layers and the high selectivity between $\mathrm{Al}_{2} \mathrm{O}_{3}$ and $\mathrm{GaAs}$ layers make the $3 \mathrm{D}$ mask ideally suited for fabricating a $3 \mathrm{D}$ photonic crystal. We could also transfer 3D photonic structures into a thick AlAs layer instead of a GaAs substrate. Then, steam oxidation can form a robust 3D mask which no longer has any shadow effects during the angle ion beam etching process.

\section{MEASUREMENT}

Our 2D photonic crystal performs as a polarizing beam splitter. The TE mode (the polarization parallel to the grooves of the grating) and TM mode (the polarization perpendicular to the grooves) of the incident light respond to the different average refractive index when passing through the photonic crystal. Therefore, if we carefully design the period and the layer thicknesses of the structure, this crystal can be used to transmit TM and to reflect TE polarized light for a broad range of incident angle and wavelength. ${ }^{14}$ This polarizing beam splitter was characterized by measuring both the transmitted and reflected power of TE and TM modes for different incident angles over a wide spectral range. Then the results were compared with numerical simulation results obtained by rigorous coupled-wave analysis (RCWA). Figure 4 shows the comparison between the measurement and simulation of the $2 \mathrm{D}$ crystal with the extinction ratio of the device as high as $820: 1$ at $1.5 \mu \mathrm{m}$.

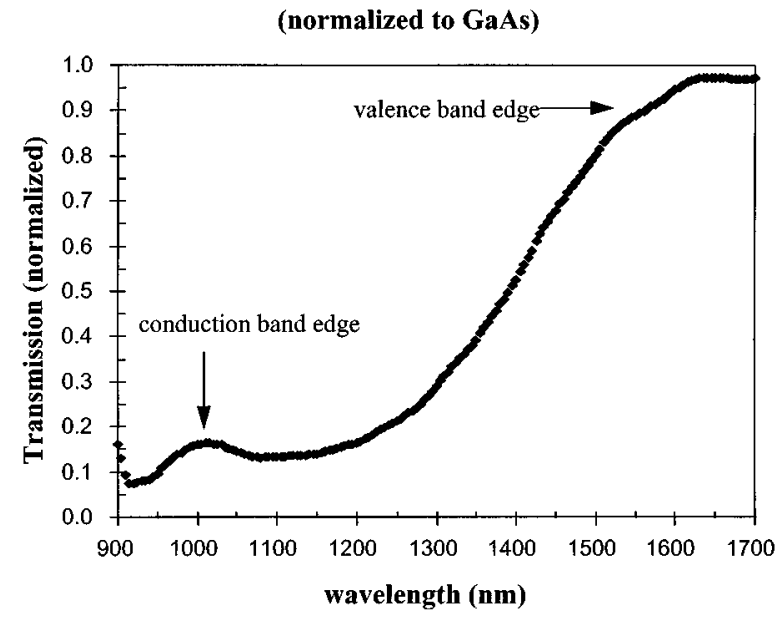

FIG. 5. Transmission spectrum shows near $90 \%$ attenuation in 3D photonic crystals. 
The transmission spectra of 3D photonic crystals are taken with a white light source passing through a monochromator, which is then focused on the crystal with a 40 $\times 40 \mu \mathrm{m}^{2}$ microfabricated $\mathrm{Ni}$ optical aperture and collected by the infrared detector. The spectrum is compared with the spectrum taken through an open aperture. Figure 5 shows a transmission spectrum of a crystal with a conduction band at $1000 \mathrm{~nm}$. The attenuation in the band gap region reaches $90 \%$ in the normal incident direction. We attribute this improvement over previous work to the use of $\mathrm{Al}_{2} \mathrm{O}_{3}$ as an etching mask which increases the number of the periods in depth.

\section{CONCLUSION}

We have shown new fabrication techniques of making high quality $2 \mathrm{D}$ and $3 \mathrm{D}$ photonic crystals by using high resolution lithography and anisotropic ion etching. A 2D photonic crystal with $600 \mathrm{~nm}$ period and $300 \mathrm{~nm}$ linewidth was fabricated by etching through $\mathrm{Si} / \mathrm{SiO}_{2}$ multilayers. It shows an extinction ratio between TE and TM modes as high as $820: 1$. The simple fabrication method used for this 2D photonic crystal lends itself to a manufacturable process. Deep 3D photonic crystals exhibit reflectivity reaching $90 \%$ in the normally incident transmission spectrum. The increase in crystal depth results from the steam oxidation of AlAs, a technique that allows the mask and the structure to be monolithically grown.

\section{ACKNOWLEDGMENTS}

The authors greatly thank Reginald Lee and Professor Amnon Yariv for supplying the samples used in this work. This research was funded by Army Research Office and National Science Foundation.

${ }^{1}$ S. John, Phys. Rev. Lett. 58, 2486 (1987).

${ }^{2}$ E. Yablonovitch, Phys. Rev. Lett. 58, 2059 (1987).

${ }^{3}$ J. D. Joannopolous, R. D. Meade, and J. N. Winn, Photonic Crystals (Princeton University, Princeton, NJ, 1995).

${ }^{4}$ E. Yablonovitch, T. J. Gmitter, and K. M. Leung, Phys. Rev. Lett. 67, 2295 (1991).

${ }^{5}$ K. M. Ho, C. T. Chan, and C. M. Soukoulis, Phys. Rev. Lett. 65, 3152 (1990).

${ }^{6}$ G. Qian and K. M. Leung, Phys. Rev. B 44, 11482 (1991)

${ }^{7}$ E. Yablonovitch, J. Opt. Soc. Am. B 10, 283 (1993).

${ }^{8}$ T. F. Krauss, R. M. De La Rue, and S. Brand, Nature (London) 383, 699 (1996).

${ }^{9}$ J. O'Brien, O. Painter, C. C. Cheng, R. Lee, A. Yariv, and A. Scherer, Electron. Lett. 32, 2243 (1996).

${ }^{10}$ C. C. Cheng and A. Scherer, J. Vac. Sci. Technol. B 13, 2696 (1995).

${ }^{11}$ C. C. Cheng, A. Scherer, V. Arbet-Engle, and E. Yablonovitch, J. Vac. Sci. Technol. B 14, 4110 (1996).

${ }^{12}$ A. Scherer, J. L. Jewell, and J. P. Haribson, Opt. Photonics News 2, 9 (1991).

${ }^{13}$ M. H. MacDougal et al., Electron. Lett. 30, 1147 (1994).

${ }^{14}$ R. C. Tyan, P. C. Sun, A. Scherer, and Y. Fainman, Opt. Lett. 21, 761 (1996). 